\title{
A study on object-oriented model repository for manufacturing system modeling
}

\author{
Yoshitaka TANIMIZU and Nobuhiro SUGIMURA \\ College of engineering, osaka prefecture university, \\ 1-1 gakuencho, sakai, osaka 599-8531, Japan, \\ phone: +81-722-54-9211, fax: +81-722-54-9904 \\ e-mail: tanimizu@syslab.mecha.osakafu-u.ac.jp \\ sugimura@syslab.mecha.osakafu-u.ac.jp
}

Kazuaki IWATA

Kochi national college of technology, 200-1 monobe, nankoku-city, kochi 783-0093, Japan, phone: +81-888-64-5500, fax: +81-888-64-5606 e-mail: piwata@jm.kochi-ct.ac.jp

\begin{abstract}
The objective of the present research is to develop a formal modeling method, aimed at realising concurrent development and integration of various manufacturing system models. An object-oriented model repository of the manufacturing system is discussed to establish a systematic and logical criteria for classifying the manufacturing system models from various viewpoints. Contents and structures of manufacturing system models are discussed from the viewpoint of the concurrent and hierarchical production planning problems, and model components and their relations to be stored in the model repository are proposed. A prototype of a model repository is developed and applied to the production planning problems.
\end{abstract}

\section{Keywords}

Modeling, manufacturing system, model repository, object-oriented approach, production planning 


\section{INTRODUCTION}

In design and planning of manufacturing systems, a wide variety of models representing the manufacturing systems are required for simulation and verification from various viewpoints. These models may be concurrently developed by different persons and/or organizations and applied to various application fields, therefore, a computerized support system is needed for concurrent development and consistency management of the various manufacturing system models.

The manufacturing system models are usually developed and applied to each application field, such as production planning of factories, production scheduling of production lines, and control of shop floors. One of the most serious problems in the modeling of the manufacturing systems is that these variety of models for the individual applications are not interrelated with each other. The problem is called islands of information (AMICE, 1992). There have been carried out various researches dealing with the methodology aiming at supporting the integration of the manufacturing system models (ISO TC184, 1898) (AMICE, 1992) (Doumeingts, 1993) (Williams, 1993) (Tanimizu, 1996).

The objective of the present research is to develop a formal modeling method, aimed at realizing concurrent development and integration of various manufacturing system models. A model repository of the manufacturing system is discussed to establish a systematic and logical criteria for classifying the manufacturing system models from various viewpoints.

Production planning are in general carried out hierarchically from the long term plans to the short term plans, and the planning tasks are sometimes carried out by different persons and/or organizations concurrently. Therefore, the model integration problem is very serious, and an effective computerized support is eagerly required. An object-oriented model repository is developed to support concurrent development and consistency management of the various manufacturing system models for production planning. Contents and structures of manufacturing system models are discussed from the viewpoint of concurrent and hierarchical production planning problems, and model components and their relations to be stored in the model repository are proposed. A prototype of a model repository is developed, and applied to the production planning problems.

\section{MODEL REPOSITORY FOR MANUFACTURING SYSTEM MODELING}

\subsection{Model generation process}

\section{Requirements for generation of various models}

One of the most significant utilization of manufacturing system models is evaluation of a real and/or virtual manufacturing system. Various types of models are generated and utilized, aimed at planning, design, implementation and control 
of the manufacturing system. The contents of the individual models depend on the items to be evaluated by using the manufacturing system models. The individual models generated are called application models, in the followings, which are suitable for the individual evaluation items. In the different application models, one facility in the manufacturing system may be represented by a set of different model components. It is necessary to provide the model builders with a computerized method to modify the contents of the model components to keep the consistency among the manufacturing system models.

In the research, a model repository is therefore proposed to generate and to utilize the models consistently. The model repository has various model components needed for generating the manufacturing system models. The relationships among the model components construct a structure in the repository. The model builders generate their own application models based on their objectives by selecting and combining the model components in the model repository as shown in Figure 1. The individual models are generated independently and concurrently, however, the generated models can be related with each other according to the relationships defined in the model repository. Therefore, a model builder is able to examine the consistency of the models with the other models generated.

\section{Hierarchy of model repository}

Various types of model components should be stored in the model repository in order to cope with the generation of various types of manufacturing systems. Therefore, multi-levels of model repositories are considered in the research; they are, generic model repository, and a set of particular model repositories. 


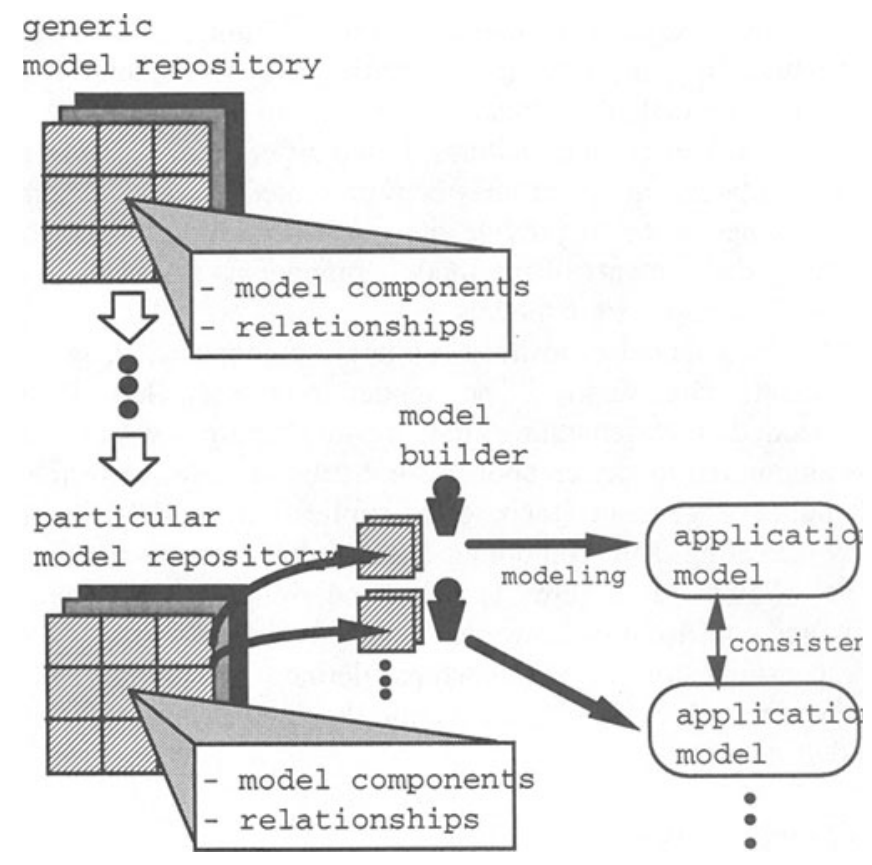

Figure 1 Model repository and modeling process of manufacturing systems

Figure 1 also shows hierarchy of the model repositories. A generic model repository of the manufacturing system model is firstly developed and implemented according to the criteria to classify the model components of the application independent manufacturing system model. A set of model components and their relationships are described and stored in the repository. Particular model repositories are secondly developed by specializing the generic model repository into a specific model repository which is suitable for the purpose and the characteristics of the manufacturing system models to be generated. Application models are thirdly generated by the model builders by combining the model components in the particular model repositories.

\subsection{Model repository}

\section{Representation of model components and their relationships}

An object oriented modeling technique is applied in the research to represent the model components and their relationships in the model repositories.

The objects representing the model components have the attributes and the activities. The attributes specify the characteristics of the components, and are described by attribute names and values. All the objects in both the generic repository and the specific repositories are class objects, and do not have any attribute values. The instances of the class objects are generated to make the 
application models. The activities specify the responses of the objects against the input to the objects. The activities affect the attribute values of only the object which has the activities and send the responses to the other objects.

The data sections of the objets are described by sets of attribute names and attribute values in the following form.

$$
\left(\mathrm{X}\left(\mathrm{p}_{1} a_{1}\right)\left(\mathrm{p}_{2} a_{2}\right) \ldots\left(\mathrm{p}_{\mathrm{n}} a_{\mathrm{n}}\right)\right)
$$

where, $\mathrm{X}, \mathrm{p}_{\mathrm{i}}$, and $a_{\mathrm{i}}$ are the object name, the attribute name and the attribute values, respectively. The activity section is given by a set of the procedural methods.

When making an application model from an particular model repository, the model builders get the model components from the model repository, arrange them according to their relationships, and give the attribute values of the model components manually or automatically to generate the instances.

\section{Classification axes of model components}

The model components which one facility in the manufacturing system is represented are examined the consistency. The class objects of these model components are classified and structured in the generic and the specific model repositories according to three kinds of axis; they are, aspect, abstraction and scope. The relationships which are used to examine the consistency among the model components are defined based on the axes. The meaning of the axes are summarised in the followings.

\section{(1) Aspect}

The aspect describes the viewpoints and the application fields of the model components. For example, a machine tool model should have an attribute of operation cost for cost management aspect. On the other hand, attributes of availability and capacity are to be included in the machine tool model for factory supervising aspect.

\section{(2) Abstraction}

The abstraction gives the degree of the abstraction of the model components. For example, a transportation machine model is an abstraction of a set of specific transportation machine models, such as AGVs, conveyors, and cranes. This axis corresponds to the is-a relations in the object-oriented model.

\section{(3) Scope}

The scope describes the extents in the space and the time of the model components. For example, a supervisor of a factory requires a model representing all the physical objects in the factory in a long time period. A model representing a status of a machine tool in a short time period is sufficient for the operator of the machine tool. This axis specifies the part-of relations in the object-oriented model. 


\section{MODEL REPOSITORY FOR HIERARCHICAL PRODUCTION PLANNING AND CONSISTENCY MANAGEMENT}

\subsection{Model components and their relations for production planning}

Production planning are in general carried out hierarchically from the long term plans to the short term plans, and the planning tasks are sometimes carried out by different persons and/or organizations concurrently. The manufacturing system models applied to the planning tasks of different levels have the different extents in the space and the time. That is, these models are classified according to the scope in the model repository shown in 2.2 .

Figure 2 shows a case where three production planning tasks are carried out concurrently and hierarchically. In the higher level, the higher level jobs called lots are loaded to the higher level resources called shops, and the production planning of the lots and the shops are carried out. In the lower level, two production planners consider the production plans of the works and the cells, which are the constituting elements of the lots and the shops, respectively. The individual production planners generate their own manufacturing system models, and determine the production plans based on the models. This model integration problem is very serious, and an effective computerized support is eagerly required. Therefore, in this research, an object-oriented model repository is developed to support concurrent development and consistency management of the various manufacturing system models for production planning.

Production planning consists of activities to select suitable manufacturing resources and their sequence for individual jobs to be manufactured, to select suitable loading sequence of the jobs to the manufacturing resources, and to determine starting time and/or finishing time of individual manufacturing operations executed by the manufacturing resources based on the pre-determined process times of the operations. 


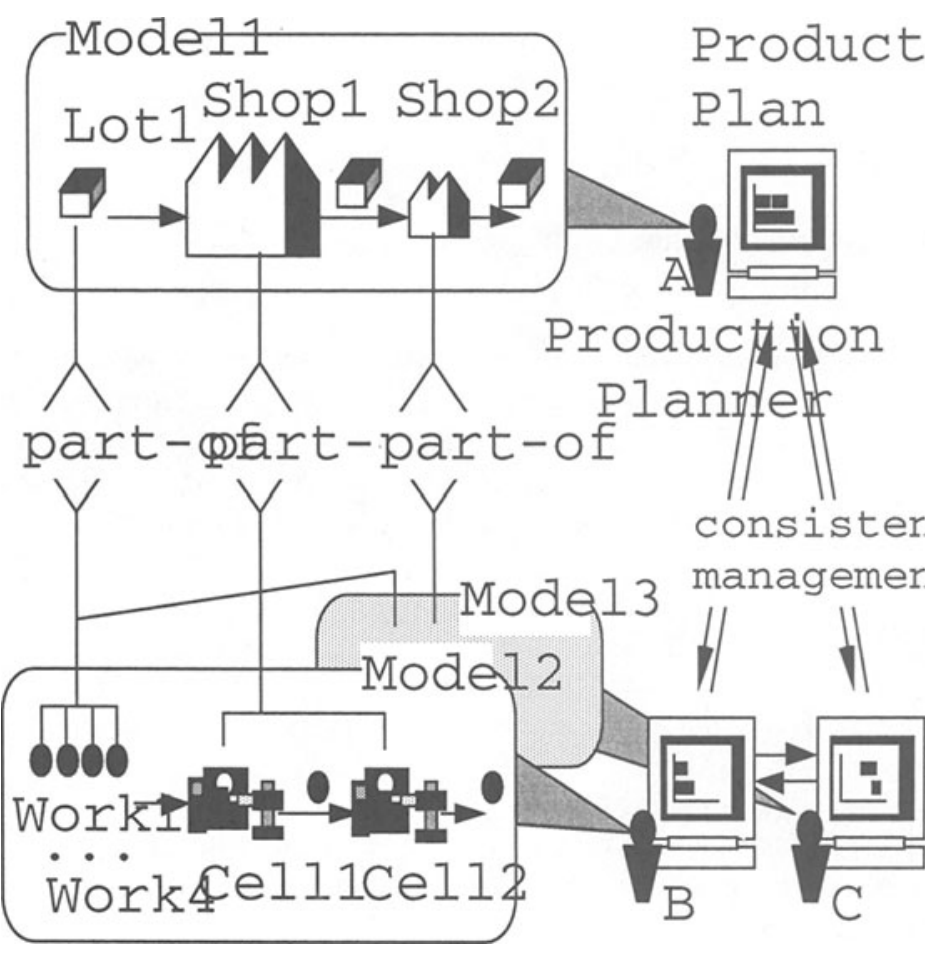

Figure 2 Concurrent and hierarchical production planning

The following objects are proposed to represent the manufacturing system models for the production planning.

(1) Job

Jobs are the objects to be manufactured in the manufacturing systems, such as the parts, the assemblies and the lots. A job has the information about the manufacturing process given by the sequence of the resources which manufacture the job. The data section of the job is described in the following form.

$$
\left(\mathrm{Jb}_{\mathrm{i}}\left(\operatorname{SeqRs}\left(\mathrm{Rs}_{1}, \mathrm{Rs}_{2}, \ldots, \mathrm{Rs}_{\mathrm{m}}\right)\right)\right.
$$

where, $\operatorname{Rs}_{\mathrm{j}}(\mathrm{j}=1, \ldots, \mathrm{m})$ are the sequences of the resources.

(2) Resource

Resources are the objects which carry out the manufacturing processes of the jobs. The machine tools, the AGVs, the manufacturing cells and the shops are examples of the resources. A resource has the information about the loading sequence of the jobs which are manufactured by the resource. The suitable loading sequence of the 
jobs is selected in the production planning tasks. The loading sequences of the resources are described in the following form.

$\left(\mathrm{Rs}_{\mathrm{j}}\left(\operatorname{SeqJb}\left(\mathrm{Jb}_{1}, \mathrm{Jb}_{2}, \ldots, \mathrm{Jb}_{\mathrm{n}}\right)\right)\right.$

where, $\mathrm{Jb}_{\mathrm{i}}(\mathrm{i}=1, \ldots, \mathrm{n})$ are the loading sequences of the jobs.

(3) Operation

Operations represent the manufacturing activities executed by the combinations of the jobs and the resources. An operation has the information about the starting time, the process time and the finishing time of the manufacturing activity. The time schedules of the individual operations are determined in the production planning tasks. The operation is described in the following form.

$\left(O \mathrm{p}_{\mathrm{ij}}(\mathrm{j} \mathrm{Jb})\left(\mathrm{rRs}_{\mathrm{j}}\right)\left(\mathrm{st}_{\mathrm{ij}} t_{1}\right)\left(\mathrm{pt}_{\mathrm{ij}} t_{2}\right)\left(\mathrm{ft}_{\mathrm{ij}} t_{3}\right)\right)$

where, $\mathrm{Jb}_{\mathrm{i}}, \mathrm{Rs}_{\mathrm{j}}, t_{1}, t_{2}$, and $t_{3}$ are the job, the resource, the starting time, the process time and the finishing time, respectively.

(4) Sequential relations among operations

Sequential relations among operations are derived from the manufacturing processes of the jobs and the loading sequences of the jobs, and given in the following form.

$\left(\mathrm{SeqOp}_{\mathrm{ij}}\left(\operatorname{prd}\left(\mathrm{Op}_{\mathrm{ij} 1}, \mathrm{Op}_{\mathrm{ij} 2}, \ldots, \mathrm{Op}_{\mathrm{ijk}}\right)\right)\left(\operatorname{suc} \mathrm{Op}_{\mathrm{ij}}\right)\right)$

where, $\mathrm{Op}_{\mathrm{ijl}}(1=1,2, ., \mathrm{k})$ are the operations to be carried out prior to the operation $\mathrm{Op}_{\mathrm{ij}}$.

When the production planning is carried out hierarchically from the long term plans to the short term plans, the manufacturing system models applied to the planning tasks of different levels have the different extents in the space and the time. That is, these models are related with each other by the part-of relations. The following three objects are needed to represent the hierarchical relations of the components of the manufacturing system models.

(5)Part-of relation among the jobs

The object gives the hierarchical relations among the jobs, and is described as follows.

$\left(\operatorname{IncJb}_{\mathrm{x}}\left(\right.\right.$ whole $\left.\mathrm{Jb}_{\mathrm{x}}\right)\left(\right.$ part $\left.\left.\left(\mathrm{Jb}_{1}, \mathrm{Jb}_{2}, \ldots, \mathrm{Jb}_{\mathrm{n}}\right)\right)\right)$

where, $\mathrm{Jb}_{\mathrm{x}}$ and $\mathrm{Jb}_{\mathrm{i}}(\mathrm{i}=1,2, \ldots, \mathrm{n})$ are the higher level job and the lower level jobs constituting the $\mathrm{Jb}_{\mathbf{x}}$, respectively. 
(6)Part-of relation among the resources

The object gives the hierarchical relations among the resources, and is described as follows.

$\left(\operatorname{IncRs} s_{y}\left(\right.\right.$ whole $\left.R s_{y}\right)\left(\right.$ part $\left.\left(R s_{1}, R s_{2}, \ldots, R s_{m}\right)\right)$

where, $R s_{y}$ and $R s_{j}(j=1,2, \ldots, m)$ are the higher level resource and the lower level resources constituting the $\mathrm{Rs}_{\mathrm{y}}$, respectively.

(7) Part-of relation among the operations

The object gives the hierarchical relations between the operations, which are derived from the part-of relations among the jobs and ones among the resources. The following shows the contents of the object.

$\left(\operatorname{IncO} p_{x y}\left(\right.\right.$ whole $\left.O p_{x y}\right)\left(p a r t\left(\mathrm{Op}_{11}, O \mathrm{p}_{12}, \ldots, O \mathrm{p}_{\text {nm }}\right)\right)$

where, $\mathrm{Op}_{\mathrm{xy}}$ and $\mathrm{Op}_{\mathrm{ij}}(\mathrm{i}=1,2, \ldots, \mathrm{n}, \mathrm{j}=1,2, \ldots, \mathrm{m})$ are the higher level operation and the lower level operations, respectively. The $\mathrm{Op}_{\mathrm{ij}}$ is a component of the $\mathrm{Op}_{\mathrm{xy}}$, if the $\mathrm{Jb}_{\mathrm{i}}$ is a component of the $\mathrm{Jb}_{\mathrm{x}}$ and the $R s_{\mathrm{j}}$ is a component of the $R s_{\mathrm{y}}$.

\subsection{Production planning process}

The production planning tasks of the individual levels are carried out through the following three steps by applying the model repository.

STEP 1: Generation of manufacturing system model

A manufacturing system model is generated by selecting and combining the model components from the repository. The instances of the jobs and the resources are generated, and the sequence of the resources is given to the jobs in order to specify the manufacturing processes.

STEP 2: Selection of loading sequence of jobs

Suitable loading sequences of the jobs are selected for the individual resources referring to the manufacturing processes. The selected loading sequences are stored in the resource objects as described in 3.1. The objects representing the operations and the sequential relations among the operations are also generated in this step.

STEP 3: Determination of starting time and finishing time of operations The starting time and the finishing time of the individual operations are determined based on the sequential relations among the operations and their process time. This task is carried out by the objects representing the sequential relations among the operations. 


\subsection{Consistency management}

In the hierarchical and concurrent production planing, the objective of the consistency management is to keep the consistency among the production plans in the different levels, which are determined concurrently and independently. In the case shown in Figure 2, the following conditions should be kept among the production plans, if the operation $O \mathrm{p}_{\mathrm{xy}}$ includes the operations $\mathrm{Op}_{\mathrm{ij}}$;

$$
\begin{aligned}
s t_{\mathrm{xy}} & =\min \left(s t_{\mathrm{ij}}\right) \\
f t_{\mathrm{xy}} & =\max \left(f t_{\mathrm{ij}}\right) \\
(\mathrm{i} & =1,2, \ldots, \mathrm{n}, \mathrm{j}=1,2, \ldots, \mathrm{m})
\end{aligned}
$$

The following equation must be satisfied among the attributes of the operations.

$$
p t_{\mathrm{xy}}=f t_{\mathrm{xy}}-s t_{\mathrm{xy}}
$$

Therefore, the following condition is derived from Equations (9), (10) and (11).

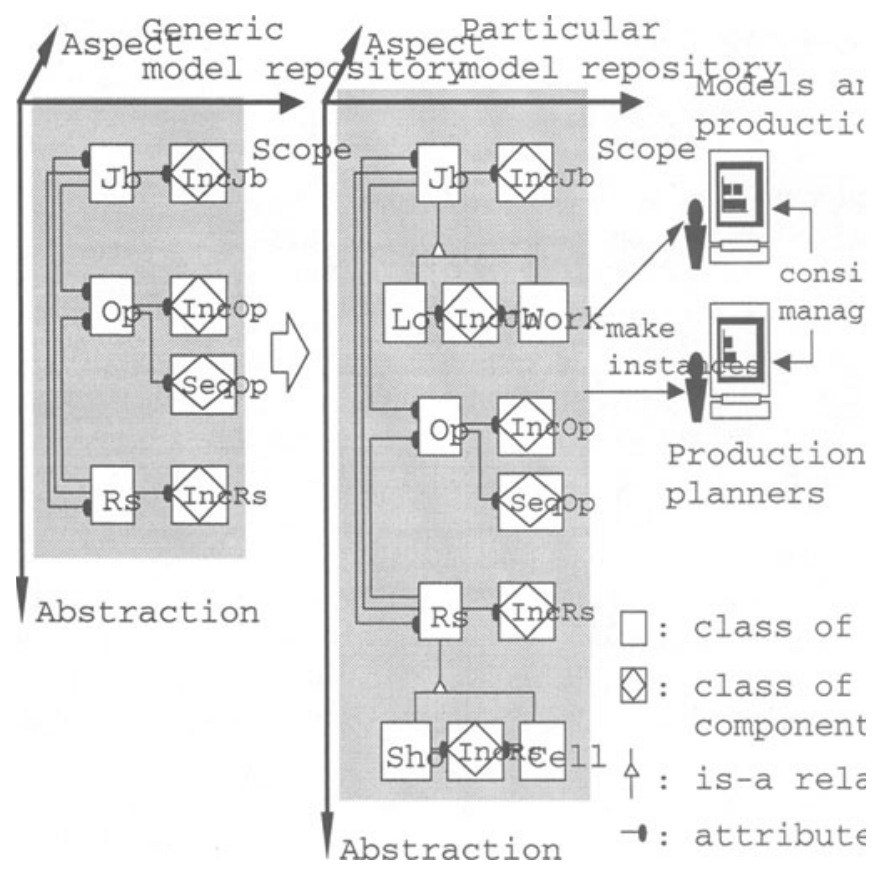

Figure 3 Model repository

$$
p t_{\mathrm{xy}}=\max \left(f_{\mathrm{ij}}\right)-\min \left(s t_{\mathrm{ij}}\right)
$$


In order to keep the conditions given by Equations (9), (10) and (12), the manufacturing system models exchange the data of the starting time and the finishing time of the operations according to the part-of relations among the operations. The production planners should change the production plans referring to the exchanged data to keep the consistency among the models.

\section{APPLICATION OF MODEL REPOSITORY}

\subsection{Model repository and modeling system}

A prototype of a model repository and a modeling system for production planning have been implemented using the object-oriented language Smalltalk. The basic architecture of the prototype system is shown Figure 3. The generic model repository for production planning stores a set of class objects mentioned in 3.1. The particular model repository for production planning is generated by making sub-class of the class objects in the generic one. The production planners generate the manufacturing system models by instantiating the class objects, and carries out the production planning based on the models.

\subsection{Case study}

A case study has been carried out to verify the effectiveness of the model repository. The manufacturing systems considered in the case study is shown in Figure 4 (a). The manufacturing system has two shops for manufacturing and painting processes. The lots consisting of sets of the works are input to and output from the shops sequentially. Therefore, the whole system is a flow shop type production system as shown in Figure 4 (b). The painting process is also a flow shop type production system where all the works are processed sequentially. The manufacturing shop consists of three manufacturing cells, and the manufacturing sequence of the individual works is different from each other. Therefore, the manufacturing shop is a job shop type production system as shown in Figure 4 (c).

It is assumed in the case study that two production planners consider the production plans for the whole manufacturing system and the ones for the manufacturing shop concurrently, and that the consistency management between the two production plans is carried out through the data exchange between the models.

Figure 5 shows an example of the hierarchical production planning tasks. Two production planners firstly generate the initial production plans shown in Figures 5 (a-1) and (b-1) which are consistent with each other. Following this, the process times of the work 2 in the manufacturing cells are increased by two times as shown in Figure (b-2) due to the same troubles in the manufacturing cells, and the change of the finishing time of the operations are sent to the manufacturing shop model. As the results, the schedules of all the operations in the manufacturing shops are 
changed automatically, and it is found that the due date of the lot 2 can not satisfy the due date as shown in Figure (a-2).

The production planner of the shops, therefore, changes the loading sequences of the lots to the shops in order to satisfy the due date. The rescheduled production plan of the shops shown in Figure (a-3) is, then, sent to the cell model in order to adjust the production plans of the cells. The modified schedules of the cells are described in Figure (b-3).

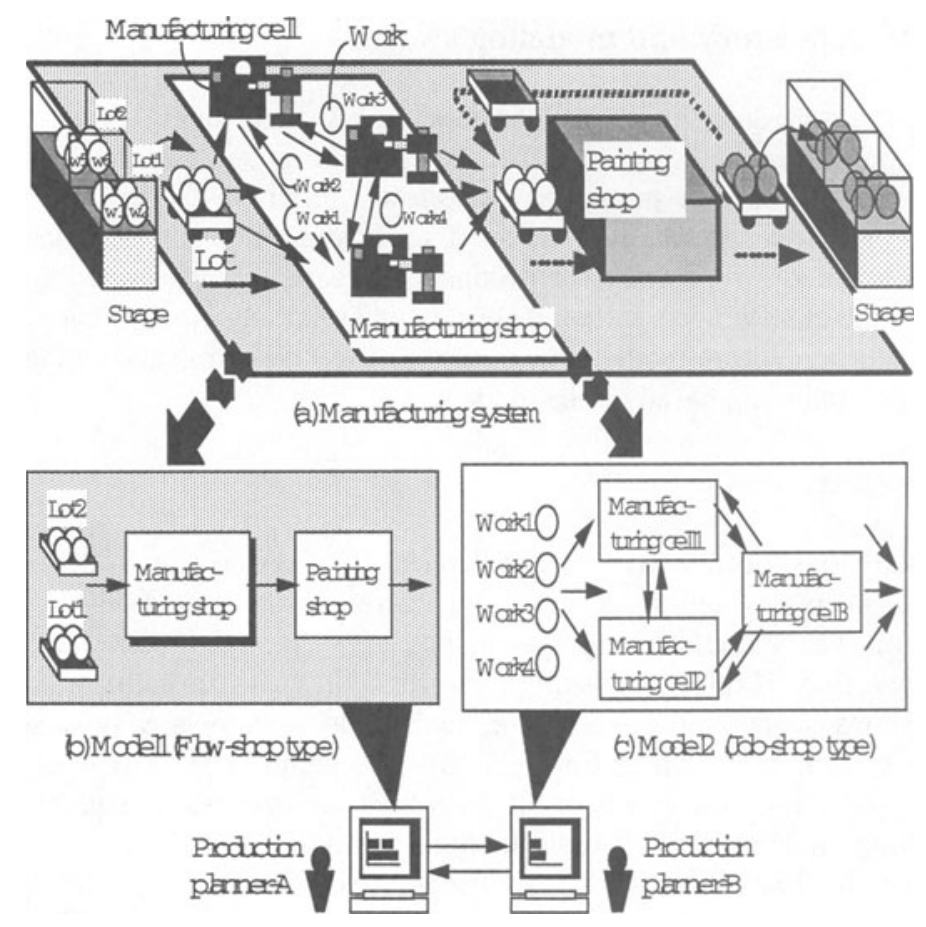

Figure 4 Target manufacturing system for case study 


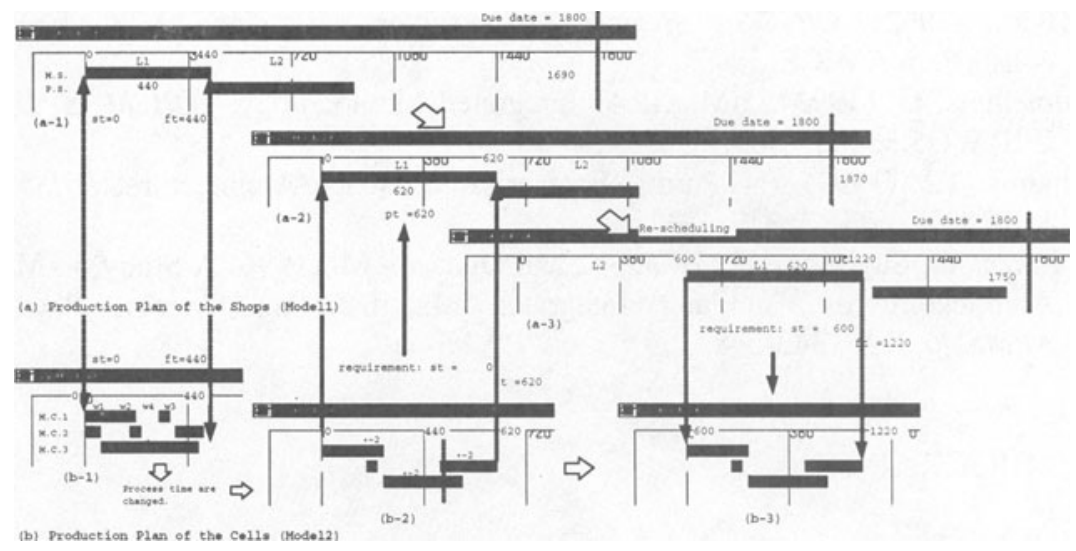

Figure 5 Example of consistency management

\section{CONCLUSIONS}

An object-oriented model repository was discussed to classify the model components of the application models for the consistency management. Concurrent and hierarchical production planning tasks were also discussed in the paper, and the proposed model repository for production planning was applied to the production planning problems. The results are summarised as follows;

- Model generation process through the model repository was proposed and multi-levels of model repositories were considered to generate various types of application models.

- Three axes representing the aspect, the abstraction and the scope were proposed to classify the model components in the model repository .

- Seven class objects were proposed as generic model components in the model repository for production planning, they are, jobs, resources, operations, sequential relations among operations, part-of relations among jobs, part-of relations among resources and part-of relations among operations. The generated production plans were kept the consistency through exchange of the information about the operations.

- A prototype system of the model repository and the modeling system was developed and applied to the concurrent and hierarchical production planning problems.

\section{REFERENCES}

ISO TC184. (1989) Reference Model for Shop Floor Production Standards, ISO TR10314. ISO TC184. 
AMICE. (1992) CIMOSA Project Deliverables Mile-stone M-2. ESPRIT consortium AMICE.

Doumeingts, G. (1993) GIM: GRAI Integrated Methodology, DIISM'93, JSPEIFIP WG5.3, 453 - 464.

Williams, T.J. (1993) The Purdue Enterprise Reference Architecture, DIISM'93, JSPE-IFIP WG5.3, 317 - 337.

Tanimizu, Y., Sugimura, N., Iwata, K. and Onosato, M. (1996) A Study on Model Architecture of Computer Integrated Manufacturing System, Proc. of APMS'96, IFIP, 343-348.

\section{BIOGRAPHY}

Yoshitaka Tanimizu received B.S. and M.S. degrees in engineering from Osaka University, Japan, in 1992 and 1994, respectively. He was a Research Associate from 1995 at College of Engineering, Osaka Prefecture University. His research interests include modeling of manufacturing systems for standardization, modeling of design process for manufacturing systems, and object-oriented modeling technique.

Nobuhiro Sugimura received B.S. and M.S. degrees in engineering from Kobe University, Japan, in 1976 and 1978, respectively. He was a Research Associate from 1980 to 1987, and a Associate Professor from 1987 to 1994 at Faculty of Engineering, Kobe University. He received a ph. D degree in engineering from Faculty of Engineering, Osaka University, in 1985. Since 1994, he is currently a Full Professor at College of Engineering, Osaka Prefecture University. His research interests include modeling and simulation of holonic manufacturing systems, CAD/CAM and product modeling for mechanical products, standardization of product model STEP, and modeling and design of machine tools.

Kazuaki Iwata graduated from Defence Academy in 1957 and received M.S. and ph. D degrees in engineering from Kyoto University, Japan, in 1961 and 1964, respectively. He was employed as a Associate Professor in 1964 and promoted to a Full Professor in 1969 at Faculty of Engineering, Kobe University. He was a Research Associate at the University of Wisconsin, Madison, USA in 1966 and 1967. He was moved to Osaka University as a Full Professor in 1989. Since 1997, he is currently at the President of Kochi National College of Technology. His research interests include advanced manufacturing systems, virtual enterprise, proficient machine system, ergonomics in manufacturing and technology forecast in future. 